\title{
Morphological variability of the palmaris longus muscle in human fetuses
}

\author{
Łukasz Olewnik $^{1}$ - Anna Waśniewska ${ }^{1} \cdot$ Michał Polguj ${ }^{3} \cdot$ Michał Podgórski $^{2} \cdot$ Piotr Łabętowicz $^{3} \cdot$ Kacper Ruzik $^{1}$. \\ Mirosław Topol ${ }^{1}$
}

Received: 13 April 2018 / Accepted: 11 July 2018 / Published online: 18 July 2018

(c) The Author(s) 2018

\begin{abstract}
Purpose The palmaris longus (PL) muscle is characterized by high-morphological variability. It is clinically important as it is routinely harvested for the reconstruction of other tendons. The study characterizes the morphology of the PL in human fetuses and creates a new classification based on its variations that would relate to the spectrum of morphological variability in adults.

Methods Eighty spontaneously aborted human foetuses (44 male, 36 female, 160 upper limbs), aged 18-38 weeks of gestation, were examined.

Results The palmaris longus muscle was present in $62.5 \%$ of fetuses. The absence was bilateral in $26.25 \%$, and unilateral in $22.5 \%$. Nine types of palmaris longus muscles were identified based on the morphology of its insertion (Types I-IX). All types originated on the medial epicondyle of the humerus. The most common type was Type I, which was characterized by insertion to the palmar aponeurosis (52\%). The rarest types were Type VII and Type IX (1\% each). Type VII was characterized by partial doubling of the muscle belly, which then turned into two separate tendons that inserted together into the palmar aponeurosis. Type IX was characterized by fusion with the flexor carpi ulnaris muscle.
\end{abstract}

Conclusion Our findings concerning morphological variability of the PL in fetuses present a new perspective on the understanding nature of the morphological variation of the PL muscle in adults.

List of evidence Basic Science Study.

Keywords Fetuses $\cdot$ Palmaris longus tendon $\cdot$ Palmaris longus muscle $\cdot$ Tendon grafts $\cdot$ Classification $\cdot$ Median nerve

\section{Abbreviations}

APB Abductor pollicis brevis

MN Median nerve

PL Palmaris longus

pd Palmar division

sd $\quad$ Second division td Third division

mpPL Muscular part of the palmaris longus muscle

PA Palmar aponeurosis

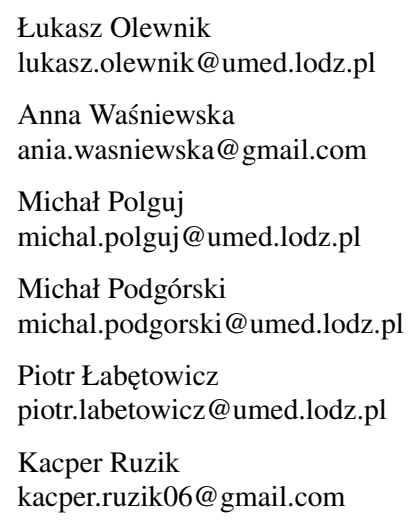

Mirosław Topol

miroslaw.topol@umed.lodz.pl

1 Department of Normal and Clinical Anatomy, Interfaculty Chair of Anatomy and Histology, Medical University of Lodz, ul. Narutowicza 60, 90-136 Lodz, Poland

2 Department of Diagnostic Imaging, Polish Mother's Memorial Hospital Research Institute, Lodz, Poland

3 Department of Angiology, Interfaculty Chair of Anatomy and Histology, Medical University of Lodz, Lodz, Poland 


\section{Introduction}

The palmaris longus (PL) is a fusiform muscle, belonging to the superficial anterior compartment of the forearm. Its initial part originates together with other superficial forearm flexors from the medial epicondyle and is covered by the anterobrachial fascia [5, 14, 21, 25]. The short belly transforms into a long, thin tendon which lies medial to the flexor carpi radialis. It passes above the flexor retinaculum and fuses with the palmar aponeurosis [6,21].

The PL is characterized by high-morphological variability including presence of an accessory muscle belly, occurrence of the reversed muscle, fusion with other muscles, the presence of an atypical tendon course, and inserts or bifurcated/multiple tendinous insertions $[1,5,7,15,18$, $20,22,25,30]$. In $1.5-63.9 \%$ of people (depending on the population) the PL may be absent unilaterally or bilaterally $[8,11,21,25,28,31]$. Morphological variation of the $\mathrm{PL}$ is clinically significant because it is commonly used as a graft for tendons transfers. For the same reason, differences in the relation of the tendon to the median nerve should be studied in order not to mismatch the two [25]. Although the PL muscle does not have a vital impact on the normal functioning of the hand, the quality of the harvested tendon might depend on the muscle and tendon variant $[4,7,10,14,15,18,20,22,25,31-33]$.

Although there have been many studies on PL in adults, knowledge about the PL in human fetuses is scare. The morphological variability of the PL in adults might result from the variety of pathways involved in its development. However, in fetuses this muscle present in more forms than in adults. Hence, the purpose of this study is to characterize the morphological variability of the PL in human fetuses and to create a new classification that would serve as a link with the spectrum of morphological variability in adults.

\section{Materials and methods}

Eighty spontaneously-aborted human fetuses (44 male, 36 female, 160 upper limbs), aged 18-38 weeks of gestation were examined. Permission for the study was given by the Local Bioethic Commission (agreement no. RNN/02/18/ $\mathrm{KE})$.

A dissection of the forearm and hand areas was performed by traditional techniques [24-27]. Firstly the PL muscle was exposed by the subcutaneous tissue and the antebrachial fascia. The morphology, together with the location and type of the insertion were evaluated. Morphometric and anthropometric measurements of the belly and tendon of the PL muscle were performed, and the distance between the midpoint of the interstyloid line (a line drawn between the styloid processes of the radius and the ulna) and the crossing of the median nerve and the PL muscle tendon were evaluated. An electronic digital caliper was used for all measurements (Mitutoyo Corporation, Kawasaki-shi, Kanagawa, Japan). Each measurement was carried out twice with an accuracy of up to $0.1 \mathrm{~mm}$.

The morphometric measurements taken from different types of PL muscle were compared using the Statistica 12.0 software package (StatSoft, Cracow, Poland). The following tests were used: the Shapiro-Wilk test was used to determine the normality of the distribution; the $\mathrm{chi}^{2}$ test and the Mann-Whitney test were used to compare nominal and contentious variables between two groups, respectively; the Kruskal-Wallis ANOVA was used to compare contentious variables between more than two groups. The level of significance was 0.05 , unless adjusted for multiple comparisons according to Bonferroni's correction.

\section{Results}

The "Results" section is divided into two parts. The first part concerns the morphology and absence of the PL, the second part describes the relationship between the tendon and the median nerve.

\section{Variation in PL morphology}

The PL was present in 100 of 160 upper limbs (62.5\%). The study recognized nine types of the PL muscle, based on variations in its form and insertion (Types I-IX). All types originated on the medial epicondyle of the humerus.

Type I originated as a muscle belly on the medial humeral epicondyle. The muscular part turned into the tendon and inserted into the palmar aponeurosis. This type was found in 52 upper limbs (52\%) (Fig. 1a).

Type II differed from Type I by the bifurcation of the distal tendon. Two components differed in size so that the 'palmar division' always predominated and inserted into the palmar aponeurosis (mean length $4.2 \mathrm{~mm}$ ), while the 'second division' was auxiliary and inserted into the flexor retinaculum of the wrist (mean length $2.44 \mathrm{~mm}$ ). The mean distance from the interstyloid line to the level of the tendon bifurcation point was $2.75 \mathrm{~mm}$. This type was observed in 22 limbs (22\%) (Fig. 1b).

Type III demonstrated a proximal attachment with the same morphology as Type I and II. The tendon was bifurcated; the 'palmar division' of the tendon always predominated and inserted into the palmar aponeurosis (mean length $3.70 \mathrm{~mm}$ ), while the 'second division' fused with the abductor pollicis brevis (mean length $2.33 \mathrm{~mm}$ ). The 
Fig. 1 Type of palmaris longus muscle. a Type I palmaris longus muscle. Left forearm. $P L$ palmaris longus muscle, $P A$ palmar aponeurosis. b Type II palmaris longus muscle. Right forearm. $P L$ palmaris longus muscle, $P A$ palmar aponeurosis, $p d$ palmar division, $s d$ second division c Type III palmaris longus muscle. $P L$ palmaris longus muscle, PA palmar division, $A P B$ abductor pollicis brevis, $p d$ palmar division, $s d$ second division. d Type IV palmaris longus muscle. $P T$ palmaris tendon, $m p P L$ muscular part palmaris longus muscle, $P A$ palmar aponeurosis

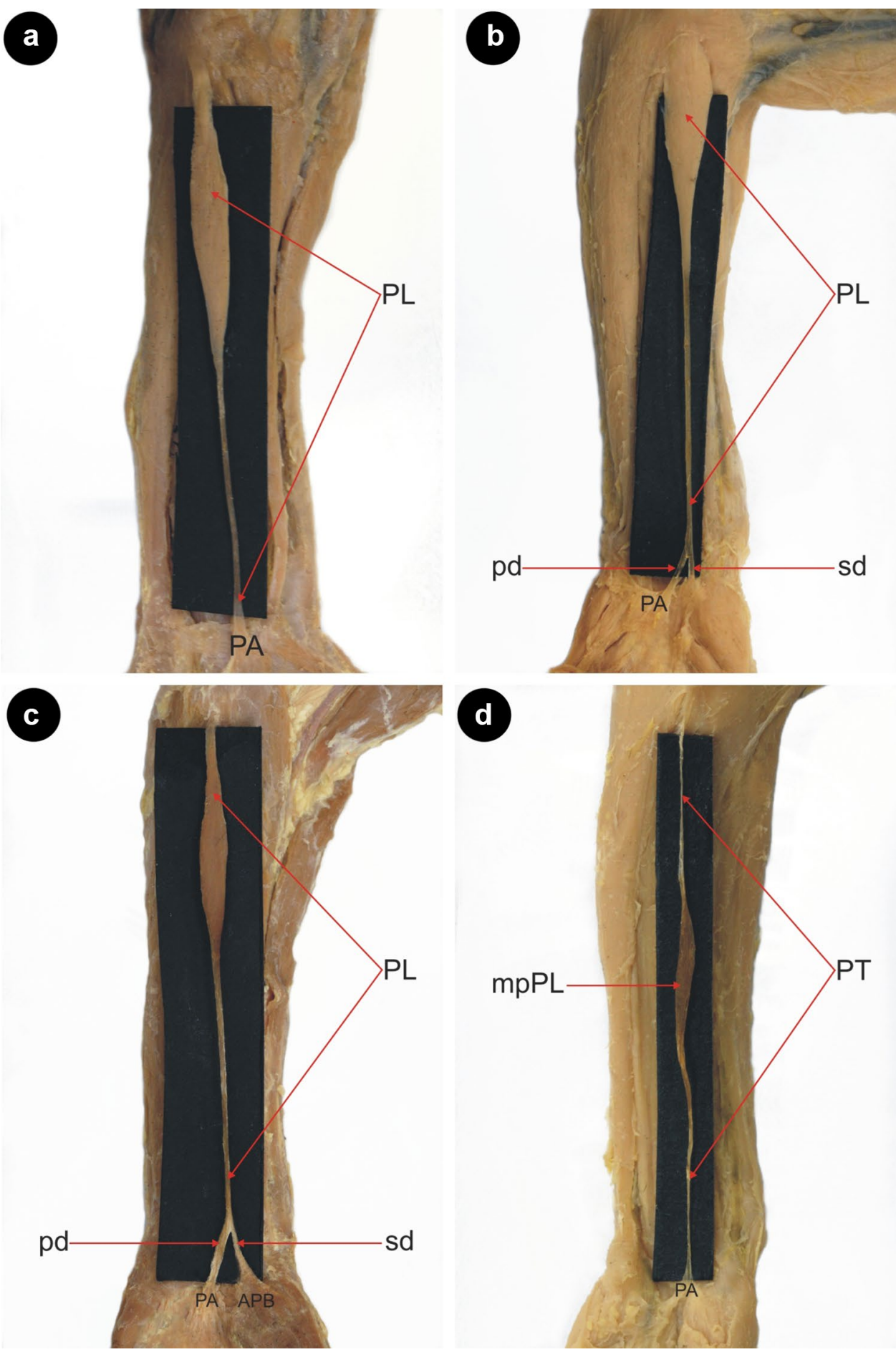

mean distance from the interstyloid line to the level of the tendon bifurcation was $1.54 \mathrm{~mm}$. This type was found in 10 cases $(10 \%)$ (Fig. 1c).

Type IV originated with a long, thin tendon from the medial epicondyle of the humerus, then the tendon gradually extended into an elongated muscle belly; slightly above the level of the interstyloid line, the muscle belly became a tendon once again, inserting into the palmar aponeurosis. This type was observed in six limbs (6\%) (Fig. 1d).

Type V demonstrated the same morphology as Type IV; however, the distal tendon was bifurcated: the 'palmar division' predominated and inserted into the palmar aponeurosis (mean length $4.26 \mathrm{~mm}$ ), while the 'second division' was auxiliary and inserted into the flexor retinaculum of the wrist (mean length $2.19 \mathrm{~mm}$ ) The mean distance from the 
interstyloid line, between the styloid process, and to the level of the tendon bifurcation was $4.08 \mathrm{~mm}$. This type occurred in three limbs (3\%) (Fig. 2a).

Type VI-this type was reversed-with the proximal portion being tendinous and distal portion being muscular. It was found in two limbs (2\%) (Fig. 2b).

Type VII-characterized by a partial doubling of the muscle belly, which then turned into two separate tendons (ulnar and radial). The length of the ulnar tendon was $7.47 \mathrm{~mm}$, and the radial tendon $9.76 \mathrm{~mm}$. The two tendons connect and inserted together into the palmar aponeurosis. This type was observed in one limb (1\%) (Fig. 2c).

Type VIII-demonstrated a proximal attachment with the same morphology as Type I, II and III. The tendon was divided into three parts: the 'palmar division' of the tendon inserted into the palmar aponeurosis (mean length $4.72 \mathrm{~mm}$ ), the 'second division' inserted into the abductor pollicis brevis (mean length $3.66 \mathrm{~mm}$ ), and the 'third division' was auxiliary and inserted into the opponens pollicis muscle (mean length $3.07 \mathrm{~mm}$ ). The mean distance from the interstyloid

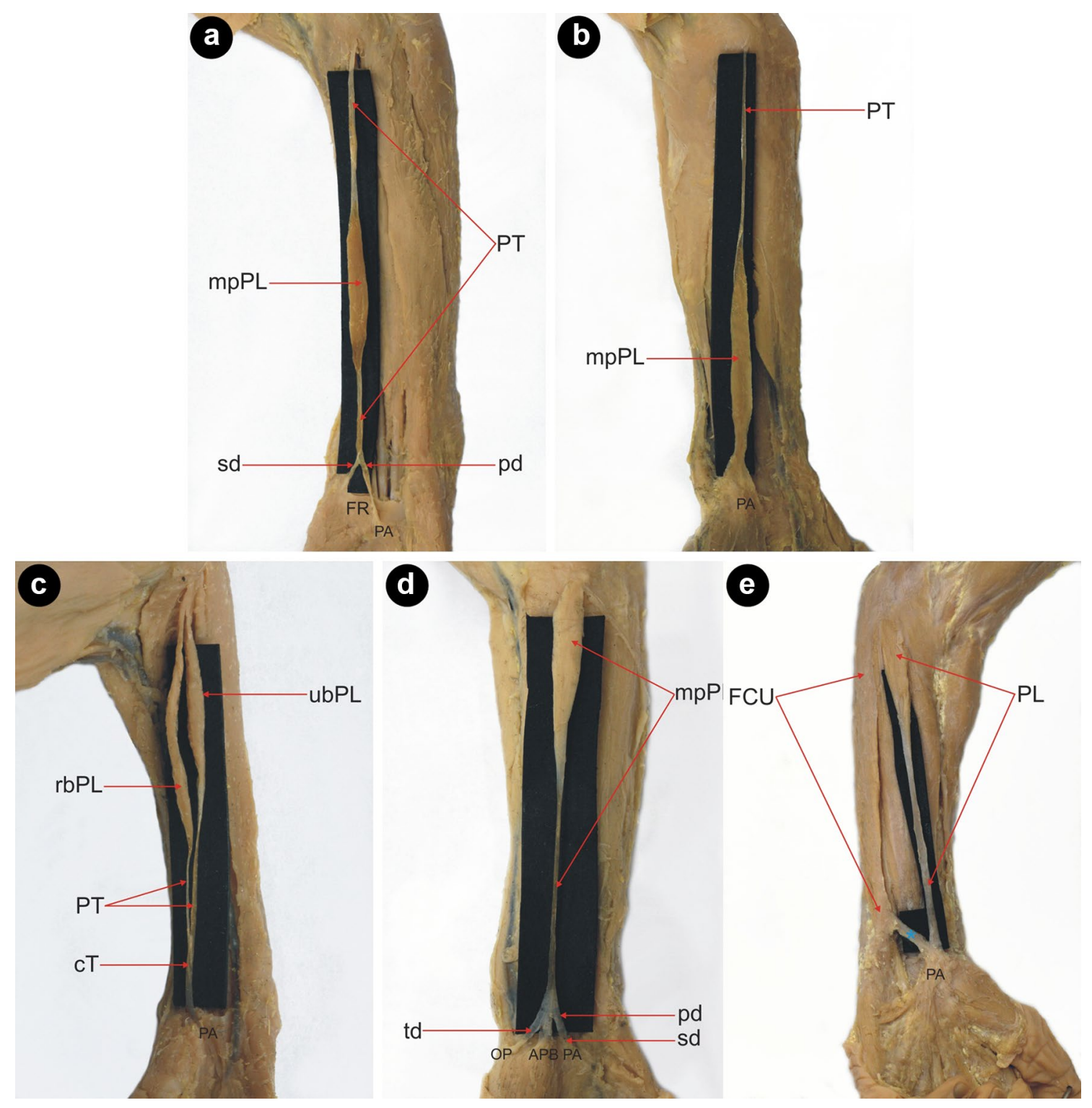

Fig. 2 Type of palmaris longus muscle. a Type $\mathrm{V}$ palmaris longus muscle. $P T$ palmaris tendon, $m p P L$ muscular part palmaris longus muscle, $P A$ palmar aponeurosis, $F R$ flexor retinaculum, $p d$ palmar division, $s d$ second division. b Type VI (reversed) palmaris longus muscle. $P T$ palmaris tendon, $m p P L$ muscular part palmaris tendon, $P A$ palmar aponeurosis. c Type VII palmaris longus muscle. $P T$ palmaris tendon, $m P P L$ muscular part palmaris longus muscle, $c T$ com- munication tendon, PA palmar aponeurosis. d Type VIII palmaris longus muscle. $m p P L$ muscular part palmaris longus muscle, $P A$ palmar aponeurosis, $A B P$ abductor pollicis brevis, $O P$ opponens pollicis muscle, $p d$ palmar division, $s d$ second division, $t d$ third division. e Type IX palmaris longus muscle. $P L$ palmaris longus muscle, $F C U$ flexor carpi ulnaris, $P A$ palmar aponeurosis $\times$ communication tendon between the palmaris tendon and flexor carpi ulnaris 
line to the level of the tendon bifurcation was $4.08 \mathrm{~mm}$. This type was found in three limbs (3\%) (Fig. 2d).

Type IX - the palmaris longus in the distal part passed above the palmar aponeurosis was connected as a "communication tendon and fused with the flexor carpi ulnaris muscle. This type was observed in one limb (1\%) (Fig. 2e).

Morphometric measurements in different types of $\mathrm{PL}$ muscle are presented in Table 1.

The PL was absent in 60 of 160 upper limbs (37.5\%). The absence was bilateral in 21 of 80 fetuses $(26.25 \%)$, and unilateral in 18 fetuses (22.5\%). The bilateral absence was more common in males (13 cases; $16.25 \%$ ) than in females (eight cases; 10\%); however, this difference was not significant $(p=0.6275)$. Unilateral absence was observed in upper limbs in nine males $(11.25 \%)$ and nine females $(11.25 \%)$ $(p=0.5786)$. Unilateral absence was observed on six right $(66.7 \%)$ and three left limbs (33.3\%) in males $(p=0.1021)$, and on seven left (77.8\%) and two right limbs (22.2\%) in females $(p=0.7658)$.

\section{Relationship between the tendon of the PL and the MN}

In this part, each type of palmaris longus tendon was checked for its relationship to the MN.

In type IV, none of tendons crossed the MN (Fig. 3b), whereas in Type II, III, VI, VII, VIII and IX all tendons crossed the MN (Fig. 3a). In type I and V, this was variable. In cases where the tendon crossed the $\mathrm{MN}$, the distance from the midportion of the interstyloid line to the point where the tendon crosses the MN was the smallest in Type VII $(2.63 \mathrm{~mm})$ and the greatest in Type VIII $(5.62 \mathrm{~mm})$. Differences between types are given in Table 2 .

In almost all types, both the mean width and mean thickness of the MN were greater than the mean width and mean thickness of the PL tendon at the cross point, except for Type VII, where the width of the PL tendon was greater than the width of the MN (Table 3).

\section{Discussion}

The PL muscle, like the plantaris muscle, is one of the most variable muscles in human body $[1,3-7,9,21-23,25-27$, 29, 32-34]. This is due to the rudimental function of this structures. More specifically, the PL muscle is well developed in species with a significantly higher ratio of upper limb weight to body weight. As this ratio is quite low in humans, i.e., the PL is less developed and its role in the functioning of the hand is reduced, it may present morphological variation [19].

With the development of the forelimb as a prehensile organ, the long flexor muscles of the forearm start to

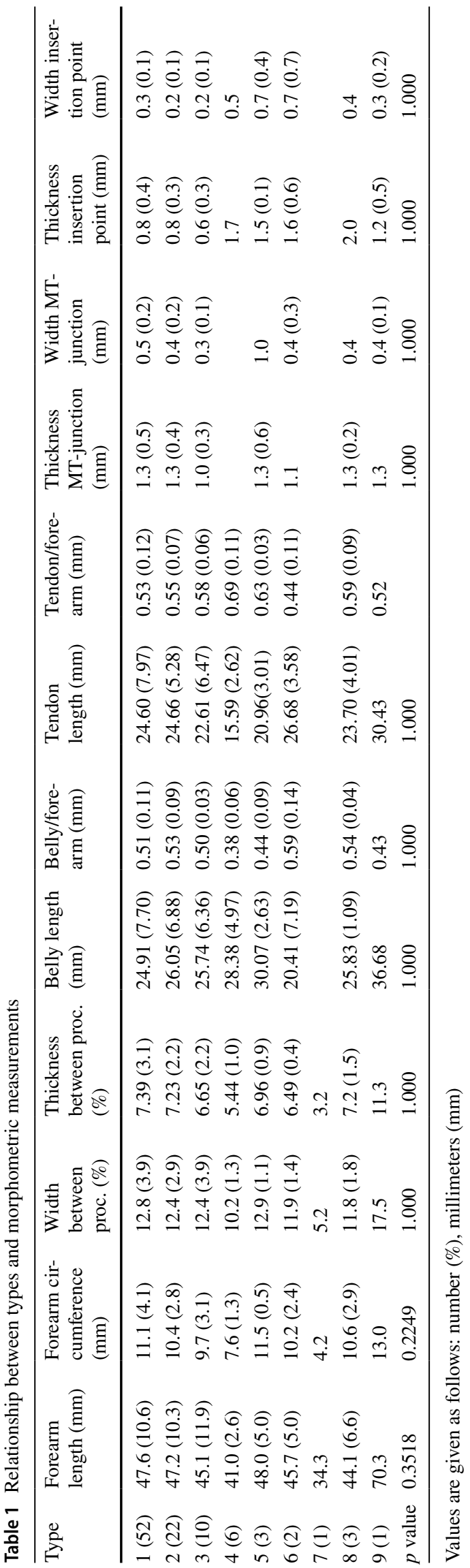


Fig. 3 Relation between the palmaris longus tendon and median nerve. a At the crossing point between the median nerve and the tendon of the palmaris longus muscle. b No crossing between the median nerve and the tendon of the palmaris longus muscle
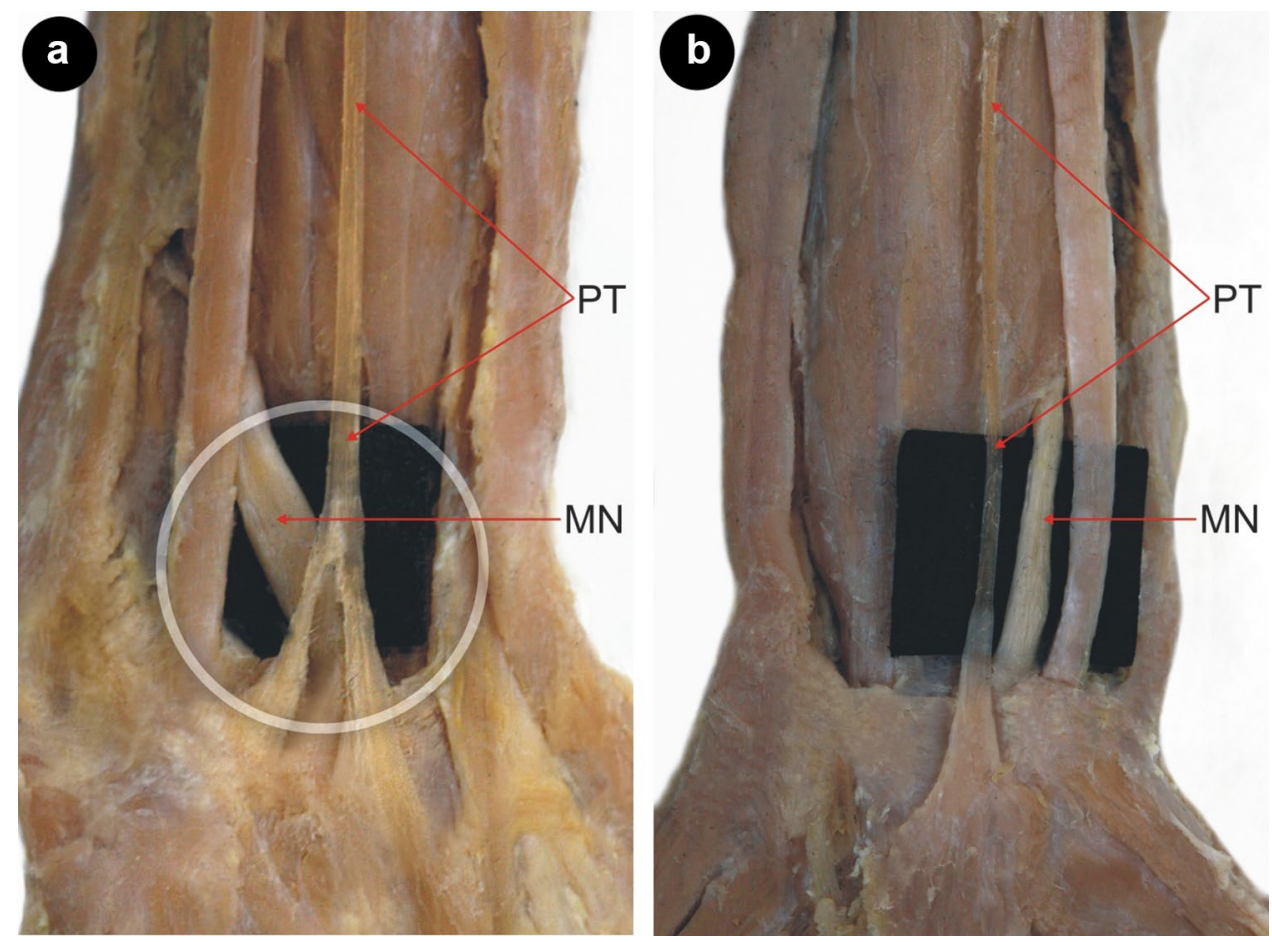

Table 2 Relationship between the types of the PL and the MN

\begin{tabular}{llll}
\hline Types & $\begin{array}{l}\text { Number of cases } \\
\text { crossing the MN }\end{array}$ & $\begin{array}{l}\text { Number of cases } \\
\text { not crossing the } \\
\text { MN }\end{array}$ & $\begin{array}{l}\text { Mean distance } \\
\text { from the mid- } \\
\text { portion of the } \\
\text { interstyloid line to } \\
\text { the point where the } \\
\text { tendon crossed the } \\
\text { MN (mm) }\end{array}$ \\
\hline I & 47 & & 4.21 \\
II & 22 & 5 & 5.61 \\
III & 10 & - & 3.90 \\
IV & - & - & - \\
V & 2 & 6 & 4.26 \\
VI & 2 & 1 & 4.28 \\
VII & 1 & - & 2.63 \\
VIII & 3 & - & 5.62 \\
IX & 1 & - & 4.36 \\
$p$ value & - & - & 0.1234 \\
\hline
\end{tabular}

partially atrophy in a caudocranial direction. These muscles develop from the flexor mass, which is further divided into superficial and the deep layers. From the deep layer arise the flexor digitorum superficialis, flexor digitorum profundus and flexor pollicis longus, while the superficial layer gives rise to the pronator teres, flexor carpi radialis and ulnaris, and the PL [19]. Among vertebrates, the palmaris longus is restricted to mammals and is most well developed in species with a weight-bearing gait. The PL is always present in Orangutans, but is variably absent in chimpanzees and gorillas. Described as one of the muscles with the most anatomical variability, it is classified as a muscle in phylogenetic regression. Morphogenetically, its tendon and muscles are developed and regulated by the HOX gene [2, 13].

Anson et al. [3] and Reinman et al. [29] presented the first major study on the anatomical variations of the PL in adults. In 1600 upper extremities, the incidence of any kind of anomaly (except for the agenesis) was 45 in 540 consecutive arms, half of which concerned variations in position and form. In the remaining half, variations included additional slips and substitute structures (15 cases), duplication of the PL (four cases) and aberrancies of attachment (three times) $[3,29]$.

Although many original studies and case reports describe morphological variants of the PL muscle in adults, very few concerned fetuses $[1,17]$. The present study distinguishes nine types of PL. Type I was the most common and was primary reported in both fetal studies $[1,17]$. Type II, with a bifurcated distal tendon, was the second most common type, occurring in $22 \%$ of cases. Albay et al. [1] also report a similar type, but while the 'second division' inserted to the flexor retinaculum in our study, it passed deep to it. Kocabiyik et al. [17] did not report this type. Surprisingly, neither authors $[1,17]$ reported variants that gave additional slips to the abductor pollicis brevis (our third most common typetype III) or that were characterized by tendon-belly-tendon morphology (our types IV and V).

On the other hand, our type VI (reversed tendon-belly morphology), identified in $2 \%$ of cases, was also described by Albay et al. [1] and Kocabiyik et al. [17]. This variant 
Table 3 Mean width and thickness at the cross point between palmaris tendon and median nerve

\begin{tabular}{lllll}
\hline Types of PL & WCMN $(\mathrm{mm})$ & TCMN $(\mathrm{mm})$ & WCP $(\mathrm{mm})$ & TCP $(\mathrm{mm})$ \\
\hline I & 0.811 & 0.244 & 1.524 & 0.772 \\
II & 0.750 & 0.234 & 1.444 & 0.620 \\
III & 0.785 & 0.189 & 1.302 & 0.585 \\
IV & - & - & - & - \\
V & 0.723 & 0.420 & 1.625 & 0.790 \\
VI & 1.120 & 0.575 & 1.710 & 0.660 \\
VII & 0.710 & 0.400 & 0.630 & 0.480 \\
VIII & 1.470 & 0.263 & 1.790 & 1.127 \\
IX & 1.150 & 0.310 & 1.330 & 1.010 \\
$p$ value & 0.2993 & 0.1353 & 0.2987 & 0.1305 \\
\hline
\end{tabular}

WCMN mean width of the tendon where the tendon crosses the median nerve, TCMN mean thickness of the tendon where the tendon crosses the median nerve, $W C P$ mean width of the median nerve at the crosses point, TCP mean thickness of the median nerve at the crosses point

is clinically significant because in adults it may predispose to median nerve compression by the muscular part of the PL [20, 22, 25]. A similar type to our Type VII (1\%), characterized by partial doubling of the belly (muscular part), was described by Albay et al. [1], although in their case the muscular part was single and only the tendon was double. Type VIII (trifurcation of the tendon; 3\% of cases) and Type IX (which was connected with flexor carpi ulnaris in distal part; $1 \%$ of cases) were not reported by Albay et al. [1] or Kocabiyik et al. [17]. Comparing the present results with those of previous studies, it can be concluded that many variations of the PL observed on the fetuses correspond to variants occurring in adults; however, Type V, VIII and IX did not $[3,4,12,14,25,29,33]$.

In adults, the PL muscle may be completely absent in between 1.5 and $63.9 \%$ of examined subjects, depending on the population.

In the present study, the PL was found to be absent in 60 limbs (37.5\%). We observed bilateral absence of the PL in $26.25 \%$ of fetuses, which is comparable with results of Albay et al. [1] (32.75\%) and Kocabiyik et al. [17] (29.2\%). More variation has been observed regarding unilateral absence, with it being reported in $22.5 \%$ of cases in the present study, $10.34 \%$ by Albay et al. [1] and $20.8 \%$ by Kacobiyik et al. [17]. For fetuses as well as for adults, the PL is more likely to be absent in female subjects and on the left side $[1,16$, 29]. One study based on an Iranian population found the PL to be absent more often in men than in women, but this difference was not significant [23]. In the present study, male and female subjects presented the same rate of unilateral absence, although it was more common for the PL to be absent on the right side in males, but on the left in females.
Due to the fact that the PL is very often used for transplants, attention should be paid to its relationship to the median nerve. One potential grafting complication is a tendon tear. The place where it tears is most likely to occur at the tendon split, this being the location where the MN crosses the tendon. Our previous study on adult identified a crossing of the MN with PL tendon in most types, except for the single case, where a fusion was observed between the palmaris longus muscle and the flexor carpi ulnaris muscle [25]. At the cross point, it was observed that the MN was wider and thicker than the PL tendon [25]; a similar observation was made in the present study for all types apart from Type VII, and no crossing was observed for Type IV. In addition, five examples of Type I and one case of Type V did not cross the MN. In our previous studies conducted on adult humans, we observed a crossing point between the PL and $\mathrm{MN}$ in all types except the type that was a fusion with flexor carpi ulnaris [25]. The present study proves that in Type IV there was no place at the crossing point between the PL and MN, while in Types I and V there were cases of non-crossing. These are the first reports on the relationship between the PL and MN. Knowledge about the variability of these structures is essential for proper planning of surgical procedures.

This study has some limitations. Due to the great variability of the PL, the proposed classification is heterogeneous and it depends on several morphological details, such as the presence of accessory division, type of origin or insertion. Nevertheless, as it was created for research purposes, it has to be detailed rather than clinically simple and immediately usable in every day practice. Secondly, we are aware the presented types and their relation to the MN may change with gestation age and after birth. However, the variability of the PL muscle in the fetus is the best starting point for analyzing its variability in adult humans.

\section{Conclusion}

Our findings shed new light on the morphological variation of the PL muscle present in fetuses. This may result in better understanding and interpretation of the variants of the PL present in adults. This is clinically significant because, despite its reduced role in the functioning of the upper limb, the PL tendon is common source of grafts for transplants.

Acknowledgements The authors wish to express their gratitude to all those who donated their bodies to medical science.

Author Contributions OŁ project development, data collection and management, data analysis and manuscript writing. WA data collection, data analysis, manuscript editing. PM data analysis, manuscript editing. PM data collection, data analysis, manuscript editing. $€ P$ data analysis, manuscript editing. RK data analysis, manuscript editing. TM 
data analysis, manuscript editing. All authors have read and approved the manuscript.

Funding There is no funding source.

\section{Compliance with ethical standards}

Conflict of interest The authors declare that they have no conflict of interest.

Ethical approval This article does not contain any studies with human participants or animals performed by any of the authors.

Open Access This article is distributed under the terms of the Creative Commons Attribution 4.0 International License (http://creativeco mmons.org/licenses/by/4.0/), which permits unrestricted use, distribution, and reproduction in any medium, provided you give appropriate credit to the original author(s) and the source, provide a link to the Creative Commons license, and indicate if changes were made.

\section{References}

1. Albay SA, Astamoni YAK, Akalli BÜŞRAS, Unali ST (2013) Anatomy and variations of palmaris longus in fetuses. Rom J Morphol Embryol 54:85-89

2. Angelini Júnior LC, Angelini FB, de Oliveira BC, Soares SA, Angelini LC, Cabral RH (2012) Use of the tendon of the palmaris longus muscle in surgical procedures: study on cadavers. Acta Ortop Bras Braz Soc Orthop Traumatol 20:226-229

3. Anson B, McVay C (1958) Surgical anatomy. W.B Sauders Company, Philadelphia

4. Aversi-Ferreira RAGMF, Bretas RV, Maior RS, Davaasuren M, Paraguassú-Chaves CA, Nishijo H, Aversi-Ferreira TA (2014) Morphometric and statistical analysis of the palmaris longus muscle in human and non-human primates. Biomed Res Int 2014:1-6

5. Barkáts N (2015) Hypertrophy of palmaris longus muscle, a rare anatomic aberration. Folia Morphol 74:262-264

6. BergmanRA,AfifiAK,Miyauchi R(2015) Illustrated Encyclopedia of Human Anatomic Variation: Opus I: Muscular System: Alphabetical Listing of Muscles. http://www.anatomyatlases.org/ AnatomicVariants/MuscularSystem/Text/P/29Palmaris.shtml. Accessed 15 Jun 2018

7. Bernardes A, Melo C, Pinheiro S (2016) A combined variation of Palmaris longus and Flexor digitorum superficialis: case report and review of literature. Morphologie 100:245-249

8. Ceyhan O, Mavt A (1997) Distribution of agenesis of palmaris longus muscle in 12 to 18 years old age groups. Indian J Med Sci $51: 156-160$

9. Dąbrowski AKP, Stankiewicz-jóźwicka H, Markuszewski M, Ciszek B (2017) The sonographic morphology of musculus palmaris longus in humans. Folia Morphol. https://doi.org/10.5603/ FM.a2017.0116

10. Erić M, Koprivčić I, Vučinić N, Radić R, Krivokuća D, Lekšan I, Selthofer R (2011) Prevalence of the palmaris longus in relation to the hand dominance. Surg Radiol Anat 33:481-484

11. Gangata $H$ (2009) The clinical surface anatomy anomalies of the palmaris longus muscle in the Black African population of Zimbabwe and a proposed new testing technique. Clin Anat 22:230-235

12. Georgiev GP, Iliev AA, Dimitrova IN, Kotov GN, Malinova LG, Landzhov BV (2017) Palmaris longus muscle variations: clinical significance and proposal of new classifications. Folia Med 59:289-297
13. Hall BK, Miyake T (2000) All for one and one for all: condensations and the initiation of skeletal development. BioEssays 22:138-147

14. Ioannis D, Anastasios K, Konstantinos N, Lazaros K, Georgios N (2015) Palmaris longus muscle's prevalence in different nations and interesting anatomical variations: review of the literature. $\mathrm{J}$ Clin Med Res 7:825-830

15. Iqbal S, Iqbal R, Iqbal F (2015) A bitendinous palmaris longus: aberrant insertions and its clinical impact-a case report. J Clin Diagn Res JCDR 9:AD03-A5

16. Kapoor SK, Tiwari A, Kumar A, Bhatia R, Tantuway V, Kapoor S (2008) Clinical relevance of palmaris longus agenesis: common anatomical aberration. Anat Sci Int 83:45-48

17. Kocabiyik N, Yildiz S, Develi S, Ozan H, Yazar F (2014) Morphometric analysis of the palmaris longus muscle: a fetal study Morphometric analysis of the palmaris longus muscle: a fetal study. Int J Exp Clin Anat 6(12):953-957

18. Kumar N, Patil J, Swamy RS, Shetty SD, Abhinitha P, Rao MK, Nayak SB (2014) Presence of multiple tendinous insertions of palmaris longus: a unique variation of a retrogressive muscle. Ethiop J Health Sci 24:175-178

19. Kumar P (2013) Duplication of palmaris longus muscle. Int J Anat Var 6:207-209

20. Marpalli S, Bhat ALS, Gadahad MRK (2016) A case of reverse palmaris longus muscle-an additional muscle in the anterior compartment of the forearm. J Clin Diagn Res JCDR 10:AD03-A4

21. Mathew AJ, Sukumaran TT, Joseph S (2015) Versatile but temperamental: a morphological study of palmaris longus in the cadaver. J Clin Diagn Res JCDR 9:AC01-A3

22. Murabit A, Gnarra M, Mohamed A (2013) Reversed palmaris longus muscle: anatomical variant-case report and literature review. Can J Plast Surg (Journal canadien de chirurgie plastique) 21:55-56

23. Nasiri E, Pourghasem M, Moladoust H (2016) The prevalence of absence of the palmaris longus muscle tendon in the north of Iran: a comparative study. Iran Red Crescent Med J Kowsar Med Inst 18:e22465

24. Olewnik Ł, Podgórski M, Polguj M, Wysiadecki G, Topol M (2017) Anatomical variations of the pronator teres muscle in a Central European population and its clinical significance. Anat Sci Int 93:299-306. https://doi.org/10.1007/s12565-017-0413-y

25. Olewnik Ł, Wysiadecki G, Polguj M, Podgórski M, Jezierski H, Topol M (2017) Anatomical variations of the palmaris longus muscle including its relation to the median nerve-a proposal for a new classification. BMC Musculoskelet Disord 18:539

26. Olewnik Ł, Wysiadecki G, Polguj M, Topol M (2017) Anatomic study suggests that the morphology of the plantaris tendon may be related to Achilles tendonitis. Surg Radiol Anat 39:69-75. https:// doi.org/10.1007/s00276-016-1682-1

27. Olewnik $Ł$, Wysiadecki G, Polguj M, Topol M (2017) The report on the co-occurrence of two different rare anatomic variations of the plantaris muscle tendon on both sides of an individual. Folia Morphol (Poland) 76:331-333

28. Raouf HA, Kader GA, Jaradat A, Dharap A, Fadel R, Salem AH (2013) Frequency of palmaris longus absence and its association with other anatomical variations in the egyptian population. Clin Anat 26:572-577

29. Reimann AF, Daseler EH, Anson BJ, Beaton LE (1944) The palmaris longus muscle and tendon. A study of 1600 extremities. Anat Rec 89:495-505 (Wiley Subscription Services, Inc., A Wiley Company)

30. Sarkar M, Mukherjee P, Roy H, Sengupta SK, Sarkar AN (2014) An unusual branch of celiac trunk feeding suprarenal gland-a case report. J Clin Diagn Res 8:AD03-4 (JCDR JCDR Research \& Publications Private Limited) 
31. Sebastin SJ, Lim AYT (2006) Clinical assessment of absence of the palmaris longus and its association with other anatomical anomalies-a Chinese population study. Ann Acad Med Singap 35:249-253

32. Sharma DK, Shukla CK, Sharma V (2012) Clinical assessment of absence of palmaris longus muscle and its association with gender, body sides, handedness and other neighboring anomalies in a population of central India. J Anat Soc India 61:13-20
33. Sunil V, Rajanna S, Gitanjali JK (2015) Variation in the insertion of the palmaris longus tendon. Singap Med J 56:e7-e9

34. Yildiz M, Sener M, Aynaci O (2000) Three-headed reversed palmaris longus muscle: a case report and review of the literature. Surg Radiol Anat SRA 22:217-219 